CU-TP-683

\title{
Vanishing Hawking Radiation from a Uniformly Accelerated Black Hole
}

\author{
Piljin Yi円 \\ Physics Department, Columbia University \\ New York, New York, 10027, U.S.A
}

\begin{abstract}
We consider quantum fields around uniformly accelerated black holes in the eternal Ernst geometry. At a particular value of the acceleration, the Bogolubov transformation which would be responsible for the late-time Hawking radiation, is found to be trivial. When this happens, Hawking's thermal radiation, Doppler-shifted or not, is absent to the asymptotic inertial observers despite the nonzero Hawking temperature, while the co-moving observers find the black hole radiance exactly balanced by the acceleration heat bath. We close with a few comments.
\end{abstract}

Physical Review Letters vol. 75 (1995) 382

PACS\#: 04.70.Dy, 03.70.+k, 04.20.Gz

\footnotetext{
${ }^{1}$ e-mail address: piljin@cuphyc.phys.columbia.edu
} 
The Hawking radiation from the black hole at rest [1], is by now a well-understood phenomenon within the semiclassical framework. Many quantum mechanical concepts, such as energy quanta and the occupation numbers, turned out to be coordinate-dependent ones, and this ambiguity leads to the particle creation in the presence of the event horizon. A black hole that has nonzero $T_{B H}$, emits thermal radiations and thereby loses its mass steadily, unless it is stabilized by a conserved local charge inside. The canonical example of the latter is given by the well-known ReissnerNordstrom (RN) black holes [2], the minimal variant of which, namely the extremal case, has zero Hawking temperature.

A related phenomenon of some interest is the so-called acceleration heat bath [3] 团. Through a similar quantum field theoretical effect as above, the usual Minkowskian vacuum feels like a heat bath to uniformly accelerated observers (sometimes called Rindler observers), with the acceleration temperature $T_{A}$ being equal to the acceleration multiplied by $\hbar / 2 \pi$.

Now a puzzling question that follows [5], is what happens to a uniformly accelerated black hole when the nonzero Hawking temperature $T_{B H}$ equals $T_{A}$. One naive expectation would be that inertial observers will find Doppler-shifted Hawking radiation (since the acceleration heat bath is not real to these observers), and that the black hole continues to evaporate. However, if one follows the black hole at large fixed distance so that he himself undergoes the same acceleration, one must find that the thermal radiation from the black hole is exactly balanced by the acceleration heat bath, and that the black hole does not evaporate at all.

One might be tempted to dismiss this puzzle [6] as a variant of the famous problem of a uniformly accelerated charge. In the latter system, co-moving Rindler observers again find a time-independent equilibrium state, yet inertial observers detect net electromagnetic radiation energy [7]. Is there a way to reconcile two such potentially conflicting observations in our case also?

Later, we will find that, unlike the case of a uniformly accelerated charge, both groups of observers actually agree in our case: No net Hawking radiation emanates from the black hole. The respective physical explanations are different, however. The Rindler observers find a co-moving heat bath that continually exchanges quanta with the black hole, in accordance with the previous expectation. On the other hand, no particle-creation (analogous to the usual Hawking radiation) occurs at all to the asymptotic inertial observers, contrary to the naive expectation. 
In order to facilitate the derivation, we will specialize to the static Ernst geometry [8], where a pair of near-extremal magnetic RN black holes are undergoing an eternal uniform acceleration. Although this enabled us to derive the desired result most clearly and succinctly, the conclusion appears quite independent of such details as whether the acceleration is eternal, in much the same way as one can derive the Bogolubov transformation relevant for Hawking's radiation in the geometry of eternal black holes. See Ref. [10] for more detail, as well as for a toy model where explicit estimates are made with more realistic initial condition.

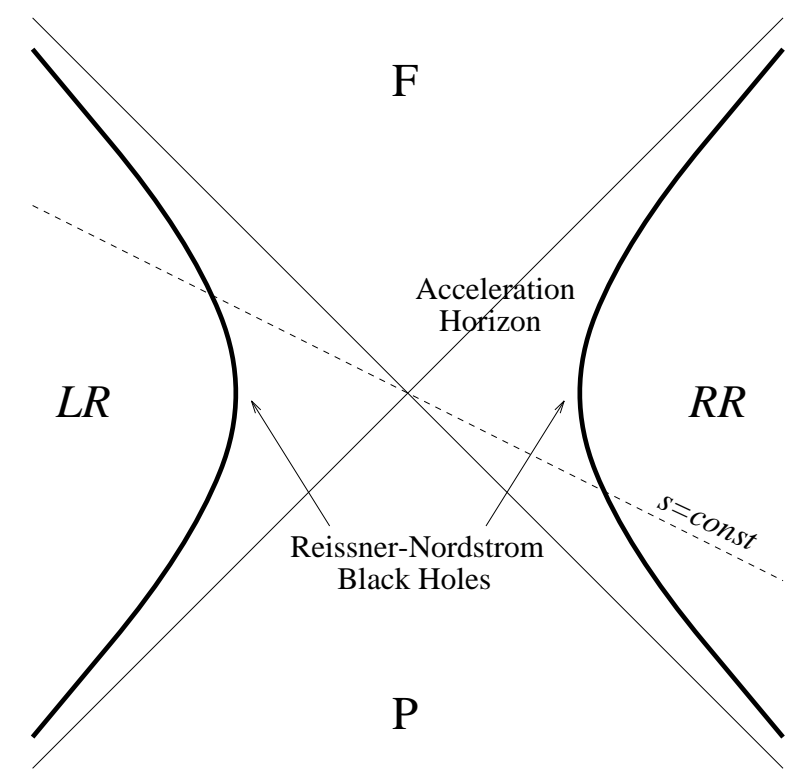

Figure 1: A schematic diagram for a pair of uniformly accelerated black holes. The black holes are represented by two hyperbolic world lines in each Rindler wedges.

Let us first write down the Ernst metric in a new coordinate system.

$$
g=\frac{\Lambda^{2}}{(1+r A x)^{2}}\left\{-F(r) d s^{2}+F(r)^{-1} d r^{2}+r^{2} G(x)^{-1} d x^{2}+r^{2} G(x) \Lambda^{-4} d \phi^{2}\right\} .
$$

The Killing time coordinate $s$ is actually a Rindler-type coordinate, so that the "static" observers are uniformly accelerated and thus, along with the black holes, must be confined within the Rindler wedges $L R$ and $R R$ in figure 1 . The spatial coordinate $r$ plays the role of the usual radial coordinate only near the black hole horizon, as is easily seen from the form of $F(r)$.

$$
F(r) \equiv-A^{2} r^{2} G(-1 / A r)=\left(1-\frac{r_{-}}{r}\right)\left(1-\frac{r_{+}}{r}-A^{2} r^{2}\right)
$$


This also shows that the geometry resembles that of the static RN black holes wherever $A r$ is sufficiently small. The external magnetic field that drives the acceleration is encoded in $\Lambda$ :

$$
\Lambda \equiv\left\{1+\frac{B x}{2} \sqrt{r_{+} r_{-}}\right\}^{2}+\frac{B^{2} r^{2}}{4(1+r A x)^{2}} G(x),
$$

where $B$ is approximately the magnetic field strength that drives the uniform acceleration.

Note that the same quartic polynomial $G$ appears in all components of the metric. Call the four roots of it, $\xi_{1}, \xi_{2}, \xi_{3}, \xi_{4}$ in the ascending order. Then, the event horizon of the black hole is at $r=\tilde{r}_{+} \equiv-1 / \xi_{2} A$, while $r=r_{A} \equiv-1 / \xi_{3} A$ is the acceleration horizon. Define the surface gravities of the horizons:

$$
\kappa_{B H} \equiv \frac{F^{\prime}\left(\tilde{r}_{+}\right)}{2}, \quad \kappa_{A} \equiv-\frac{F^{\prime}\left(r_{A}\right)}{2}
$$

which are related to the temperatures by $T_{B H}=\hbar \kappa_{B H} / 2 \pi$ and by $T_{A}=\hbar \kappa_{A} / 2 \pi$. When the size of the black holes are relatively small $\left(r_{ \pm} A \ll 1\right), A \simeq \kappa_{A}$ can be regarded as the acceleration of the black hole.

Since we are interested in the cases where the co-moving Rindler observers find a complete thermal equilibrium, we want to require that the Hawking temperature be equal to the acceleration temperature. In terms of the surface gravities, therefore, we demand that

$$
\kappa \equiv \kappa_{B H}=\kappa_{A}
$$

In some cases, most notably when the black hole mass is much larger that its charge, $\kappa_{B H}>\kappa_{A}$ is always true and this constraint can never be met. However, when the non-extremal RN black holes in question are sufficiently close to the extremality, it is possible to achieve this fine-tuning [5]. In fact, this constraint is naturally imposed if the two black holes are pair-created via the wormhole-type instanton [9].

In addition to the above considerations, it is most essential for our purpose that we understand the causal structure of the space-time. First of all, recall that the acceleration horizon divides the asymptotic infinities into two groups: ones inside Rindler wedges and ones outside. Inside the Rindler wedges $L R$ and $R R$, there are asymptotic null or space-like infinities that correspond to $x=\xi_{3}=-1 / A r$. However, most spacetime trajectories of energy quanta, being either time-like or null, will eventually cross acceleration horizon into the region $\mathrm{F}$ which is inaccessible to those 
"static" Rindler observers [7]. In order to remain within the Rindler wedge forever, the particle must either have magnetic charge or be directed exactly parallel to the axis of the uniform acceleration. It follows that in order to understand the radiative process, one may safely ignore the infinities in the Rindler wedges. The resulting causal structure can be found in figure 2 below. Throughout this letter, we shall assume that there exist no "light" magnetically charged particles.

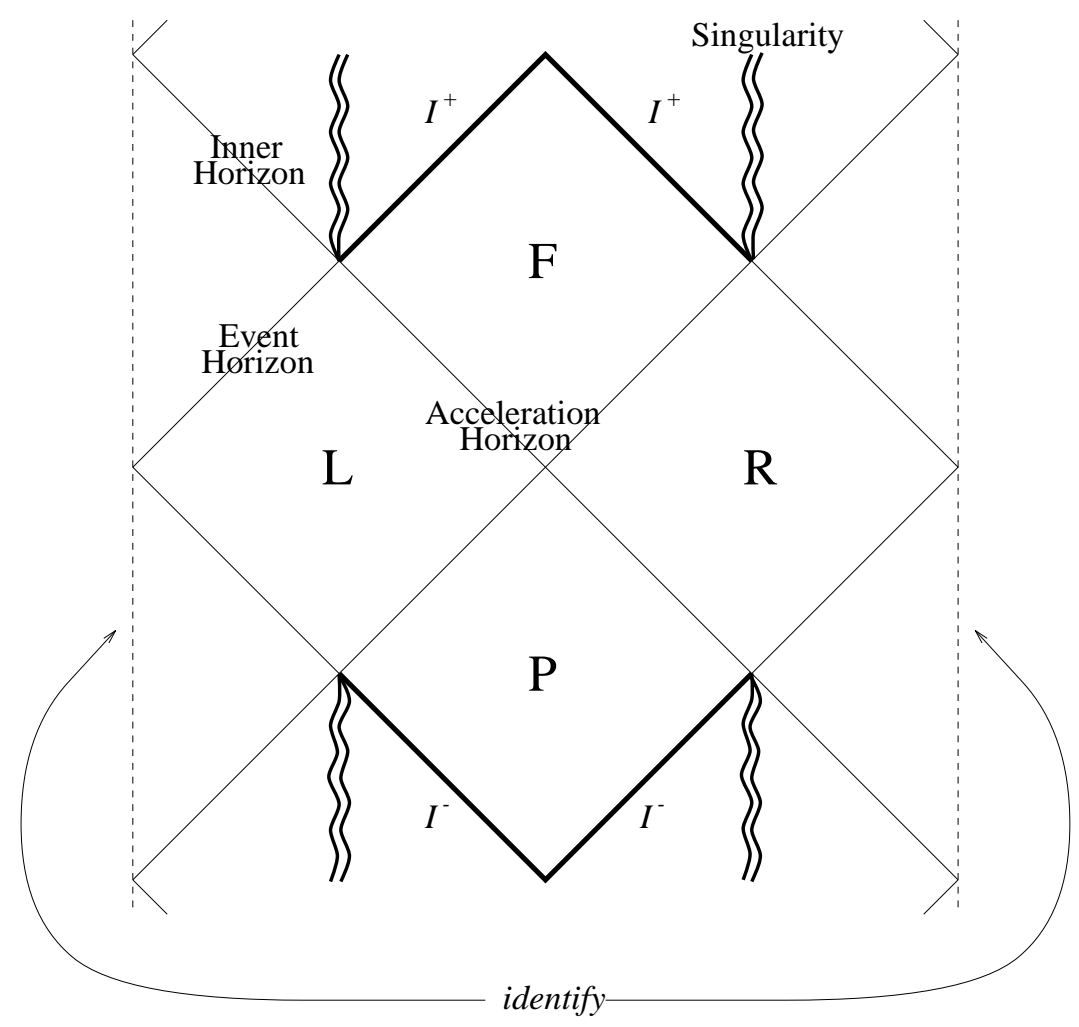

Figure 2: Penrose diagram of the Ernst spacetime with the Rindler infinities at $x=\xi_{3}=$ $-1 / A r$ excised. The bold (straight) lines indicate the asymptotic infinities.

This spacetime actually represents a wormhole. In figure 2 , the left end of the diagram is identified with the right end. However, this wormhole is different from those found in eternal black holes [2] in one important aspect: Here, the two mouths (or equivalently the two black holes) share the same asymptotic future F.

As a first step in quantizing matter fields, consider the field equation for a free scalar in regions 
$\mathrm{L}$ and R. For this purpose, it is most convenient to introduce a new tortoise-like coordinate $z$ between the two horizons $\left(\tilde{r}_{+} \leq r \leq r_{A}\right)$ :

$$
z \equiv \int^{r} d \tilde{r} \frac{1}{F(\tilde{r})}
$$

which logarithmically approaches $-\infty$ at the event horizon and $+\infty$ at the acceleration horizon. After rescaling the eigenmodes $\Psi^{(w, m)}$, for each Rindler frequency $w>0$ and the quantized angular momentum $m$,

$$
\Psi^{(w, m)}=e^{\mp i w s} \frac{(1+r A x)}{r}\left[\Phi_{(w, m)}(r, x) e^{i m \phi}\right]
$$

we find the following equation that must be solved for the eigenmodes:

$$
w^{2} \Phi_{(w, m)}+\frac{\partial^{2}}{\partial z^{2}} \Phi_{(w, m)}=F(r(z))\left\{\frac{1}{r^{2}}\left[-\frac{\partial}{\partial x} G(x) \frac{\partial}{\partial x}+\frac{m^{2} \Lambda^{4}}{G(x)}\right]+U_{\mathrm{eff}}\right\} \Phi_{(w, m)} .
$$

$U_{\text {eff }}$ is a bounded function of $z$ and $x$, and in particular contains the mass term and possible curvature couplings. Note that the right-hand-side of Eq. (8) has the overall factor $F(r(z))$ that vanishes exponentially $\sim e^{-2 \kappa|z|}$ as $|z| \rightarrow \infty$. In the same limit, $\Lambda$ is reduced to functions of $x$ only, $\Lambda_{A} \equiv \Lambda\left(r=r_{A}\right)$ or $\Lambda_{B H} \equiv \Lambda\left(r=\tilde{r}_{+}\right)$.

Introducing two null coordinates $u=s-z$ and $v=s+z$, we find the following general behavior, near each horizon, of the future-directed Rindler eigenmodes $\Psi_{L}^{(w, m)}$ and $\Psi_{R}^{(w, m)}$ that have respective supports in either $\mathrm{L}$ or $\mathrm{R}$,

$$
\begin{array}{lllll}
\Psi_{L}^{(w, m)} \sim e^{-i w u} C_{\lambda m}(x) e^{i m \phi} & \text { or } e^{-i w v} C_{\lambda m}(x) e^{i m \phi} & \text { in } \mathrm{L}, & \Psi_{L}^{(w, m)}=0 & \text { in } \mathrm{R}, \\
\Psi_{R}^{(w, m)} \sim e^{+i w u} C_{\lambda m}(x) e^{i m \phi} & \text { or } e^{+i w v} C_{\lambda m}(x) e^{i m \phi} & \text { in } \mathrm{R}, & \Psi_{R}^{(w, m)}=0 & \text { in } \mathrm{L} .
\end{array}
$$

The positive sign in (10) is because $(u, v)$ grow toward past rather than future in the region $\mathrm{R}$. The same set of symbols $C_{\lambda m}$ and $\lambda$ are used to denote eigenfunctions and eigevalues for two different eigenvalue problems, the relevant operators of which are obtained from the one inside the square bracket in Eq. (8), by replacing $\Lambda$ by $\Lambda_{A}$ or by $\Lambda_{B H}$. In particular, due to the lack of the spherically symmetry, an eigenmode that has a definite $\lambda$ near the event horizon will not have a definite $\lambda^{\prime}$ near the acceleration horizon. Similarly, the backscattering effect will mix the left-moving modes with the right-moving modes. But since none of these details matter, as we will find out shortly, we shall keep just one superscript $w$ form now on. 
Let us be reminded that, for each positive Rindler mode $\Psi^{(w)}$ with $w>0$, there exists a timereversed negative mode $\Psi^{(-w)}$ that propagates backward but otherwise of the same form: The complete Hilbert space is spanned by both positive and negative modes. But the point is, such labels as future-directed and past-directed are inherently observer-dependent. A purely futuredirected (positive) mode in one coordinate system could be a mixture of both future-directed (positive) and past-directed (negative) modes as perceived in another. Accordingly, the so-called Bogolubov transformation, which maps one basis to the other, is such that a vacuum with respect to one set of observers can actually be an excited state with respect to the other. And this is exactly the origin of both the Hawking radiation and the acceleration heat baths [1] [4].

In this regard, it is important to realize that $(u, v)$ are not good coordinates for inertial observers and must be traded off in favor of Kruskal-type coordinates that play the role of advanced and retarded times for local inertial observers. The approximate form of such coordinates near the respective horizons, are completely determined by the surface gravity $\kappa$ alone: Calling the Kruskal coordinates near the event horizon $\left(U_{1}, V_{1}\right)$, we find,

$$
\begin{aligned}
& \kappa u \simeq-\ln \left(-U_{1}\right) \quad \text { in } \mathrm{L}, \quad \kappa u \simeq-\ln \left(+U_{1}\right) \quad \text { in } \mathrm{R}, \\
& \kappa v \simeq+\ln \left(+V_{1}\right) \quad \text { in } \mathrm{L}, \quad \kappa v \simeq+\ln \left(-V_{1}\right) \quad \text { in } \mathrm{R} .
\end{aligned}
$$

For the other Kruskal coordinates $\left(U_{2}, V_{2}\right)$ near the acceleration horizon, we simply replace $\left(U_{1}, V_{1}\right)$ by $\left(U_{2}, V_{2}\right)$ and reverse every single sign on the right-hand-side.

At last, we are ready to obtain the eigenmodes of positive frequencies with respect to inertial observers. For the purpose, we may use Unruh's characterization of positive frequency [4]: a simple analyticity argument shows that a positive frequency mode must be analytic and bounded in the lower-half-plane of the complexified time coordinate. For instance, a positive frequency mode as detected by the inertial observers near the event horizon, must be analytic in $U_{1}$ and $V_{1}$ throughout their lower-half-planes.

Since the Rindler modes are defined in either L or R, they are defined only on the half-lines of Kruskal coordinates. To construct the eigenmodes that are appropriate for inertial observers, one expresses $\Psi_{L}^{(w)}$ and $\Psi_{R}^{(w)}$ in terms of $\left(U_{i}, V_{i}\right)$ for $i=1,2$ using the coordinate transformations above in (11) and (12), and analytically continue the logarithms through lower-half-planes of each Kruskal coordinates. Then the resulting modes have positive frequencies with respect to inertial observers, 
in addition to having the supports on the entire spans of Kruskal coordinates. For inertial observers near the event horizon of the black holes, the positive frequency modes $\Psi_{B}^{(w)}$ are

$$
\Psi_{B L}^{(w)} \simeq N_{w}\left[\Psi_{L}^{(w)}+e^{-\pi w / \kappa} \Psi_{R}^{(-w)}\right], \quad \Psi_{B R}^{(w)} \simeq N_{w}\left[\Psi_{R}^{(w)}+e^{-\pi w / \kappa} \Psi_{L}^{(-w)}\right]
$$

where $N_{w} \equiv 1 / \sqrt{1-e^{-2 \pi w / \kappa}}$. The expressions for $\Psi_{B}^{(-w)}$ can be obtained similarly.

For an ordinary nonaccelerated black holes $\left(\kappa=\kappa_{B H}, \kappa_{A}=0\right)$, this would be the end of the story, since the Rindler coordinates $(u, v)$ are in that case the ordinary retarded and advanced times asymptotically: the positive Rindler modes $\Psi^{(w)}$ themselves are future-directed with respect to asymptotic inertial observers. For physical black holes with smooth future event horizon, then, the physical vacua are such that the asymptotic inertial observers will find outward thermal radiation at $\left.T_{B H}=\hbar \kappa_{B H} / 2 \pi \llbracket 1\right]$.

However, with the uniformly accelerated black hole, $(u, v)$ are not good asymptotic inertial coordinates. Rather, $\left(U_{2}, V_{2}\right)$ are. Far away from the black hole, the metric (11) is essentially that of the Melvin spacetime in a Rindler-type coordinate system [6], which means that $\left(V_{2}+U_{2}\right) / 2$ may be regarded as the asymptotic Minkowski time. This is particularly clear when the black holes are relatively small $\left(r_{ \pm} A \ll 1\right)$ [5].

We must perform another Bogolubov transformation and superimpose it to the one in Eq. (13). Near the acceleration horizon, the situation is identical to the above (because $\kappa_{A}=\kappa \equiv \kappa_{B H}$ ), except that the relative positions of $\mathrm{L}$ and $\mathrm{R}$ are switched. Calling the asymptotic inertial modes $\Psi_{A}^{(w)}$, s, we find near the acceleration horizon:

$$
\Psi_{A R}^{(w)} \simeq N_{w}\left[\Psi_{R}^{(w)}+e^{-\pi w / \kappa} \Psi_{L}^{(-w)}\right], \quad \Psi_{A L}^{(w)} \simeq N_{w}\left[\Psi_{L}^{(w)}+e^{-\pi w / \kappa} \Psi_{R}^{(-w)}\right]
$$

Combining the two transformations in (13) and (14), we finally find that the Bogolubov transformation between the two groups of inertial observers, is actually trivial. That is, it does not mix positive and negative frequency modes:

$$
\Psi_{B}^{(w)} \Rightarrow \Psi_{A}^{(w)}, \quad \Psi_{B}^{(-w)} \Rightarrow \Psi_{A}^{(-w)}
$$

As was noted earlier, the respective forms of the Rindler modes near each horizon must be taken with a grain of salt: Combining the two Bogolubov transformations, in general, we must include 
a unitary transformation $\mathcal{U}$ that reflects the effects of local geometry between the two horizons. On the other hand, the Rindler time $s$ is a Killing coordinate, and $\mathcal{U}$ has to commute with the Rindler energy operator $i \hbar \partial_{s}$. Hence, with the Bogolubov transformations above that depend only on the Rindler frequency $w$, this additional complication cannot alter our conclusion: we find that no late-time Hawking radiation reaches the asymptotic inertial observers.

This may be compared to the classic puzzle of the Bremmstrahlung from a uniformly accelerated charge. There too, the energy of the moving charge is not diminished in any way, thanks to the vanishing radiation backreaction, yet asymptotic inertial observers find classical electromagnetic radiation emanating from the charge. This energetics part of the puzzle was first resolved by Coleman [11] in part and then later by Boulware [7] more completely, who observed that the radiation actually originates from the conversion of the "pre-acceleration" Coulomb field to the one associated with the moving charge. If the uniform acceleration is terminated in the future, the charge will give up some of its kinetic energy to restore the Coulomb field again, but as long as the uniform acceleration is maintained, all the radiation energy is derived from the continuously varying electromagnetic field around the charge. The obvious difficulty anyone must face in trying to find a similar mechanism in our problem, is simply that there is no such "pre-acceleration" field for each and every particle species: the Hawking radiation is completely universal in its composition.

We would like to close with a few comments. First, we wish to emphasize that the so-called late-time approximation is employed here, as in most derivations of black hole radiance. While the main result (15) precludes any analogue of the late-time Hawking radiation, we still may not be able to account for possible (sub-leading) transient behaviors. In fact, such an effect that corrects the black hole mass by a finite and small factor $\sim \hbar / r_{+}^{2}$, was previously observed for static extremal RN black hole [12] directly as well as for pair-produced near-extremal RN black holes [5] indirectly. However, once a steady state (with $\kappa_{A}=\kappa_{B H}$ ) is reached, the main result (15) tells us that no further radiation escapes into the asymptotic future.

Second, we must point out that, although our method can be used to derive the nontrivial Bogolubov transformation when $\kappa_{A} \neq \kappa_{B H}$, it does not automatically provide the spectrum that the asymptotic inertial observers detects. One of the reason is, the eigenmodes $\Psi_{A}^{(w)}$ have a definite 
absolute value of energy (although not the sign thereof) with respect to the Rindler time $s$, not with respect to the the asymptotic inertial time $\left(V_{2}+U_{2}\right) / 2$. (In our derivations above, however, this fact was actually to our advantage, for it enabled us to circumvent nasty complications that may arise from the Doppler effect.) More direct approaches may be desirable.

The author is grateful to Jaemo Park, J. Preskill and S. Trivedi for interesting conversations at an early stage of this work. He also thanks Kimyeong Lee, S.A. Ridgeway and E. Weinberg for reading a preliminary version of the manuscript. This work is supported in part by U.S. Department of Energy.

\section{References}

[1] S.W. Hawking, Comm. Math. Phys. 43 (1975) 199.

[2] See S.W. Hawking and G.F.R. Ellis, The large scale structure of space-time, Cambridge University Press, 1973.

[3] S.A. Fulling, Phys. Rev. D7 (1973) 2850; P.C.W. Davies, J. Phys. A8 (1975) 609.

[4] W.G. Unruh, Phys. Rev. D14 (1976) 870.

[5] P. Yi, Phys. Rev. D51 (1995) 2813.

[6] F. Dowker, J.P. Gauntlett, S.B. Giddings, G.T. Horowitz, Phys. Rev. D50 (1994) 2662.

[7] D.G. Boulware, Ann. Phys. (New York) 124 (1980) 169; A. Higuchi, G.E.A. Matsas and D. Sudarsky, Phys. Rev. D45 (1992) R3308.

[8] F.J. Ernst, J. Math. Phys. 17 (1976) 515.

[9] D. Garfinkle and A. Strominger, Phys. Lett. 256B (1991) 146.

[10] P. Yi, Quantum Stability of Accelerated Black Hole, CU-TP-690, hep-th/9505021.

[11] S. Coleman, Rand Report RM-2820 (1961), unpublished.

[12] J. Park and P. Yi, Phys. Lett. 317B (1993) 41. 